\title{
Perturbation of Temperature Fields by a Small Inclusion.
}

\author{
D. GöHDE
}

In einem Gebiet, das ein kleines Teilgebiet $\dot{\Omega}_{a}$ enthält, wird ein Randwertproblem zweiter Ordnùng mit Kopplungsbedingungen auf dem Rand von $\Omega_{\varepsilon}$ gestellt. Für seine lösung wird eine asymptotische Entwicklung bezüglich des nach 0 gehenden Durchmessers von $\Omega_{8}$ konstruiert.

В области содержащей малую подобласть ' $\Omega_{\varepsilon}$ ставится краевая задача́ второго порндка с переходным, условием на границе $\Omega_{\varepsilon}$. Для её решения строится асимптотика относительно диаметра $\Omega$; :стремящегося к нулю.

In a domain which contains a small subdomain $\Omega_{\varepsilon}$ a boundary value problem of second order with transition conditions on the boundary of $\Omega_{\mathrm{t}}$ is posed. For its solution an asymptotic expansion is constructed with respect to the diameter of $\Omega_{\varepsilon}$ tending to zero.

For the last decades, several phenomena in physical science and engineering have - given raise to models of mathematical analysis which are concerned with domains with irregular boundaries (e.g., porous media or perforated walls) and therefore, especially since about ten years, to a literature referring to this. Within this topic, it seems $A$. M. Il'in had been the'first to investigatè, to some extent, a fundamental type of problems' namely; boundary value problems in domains with one small hole: From a bounded domain $G$, a small subset $\Omega_{\varepsilon}$ of diameter $\varepsilon$ is removed, and the solution of a boundary value problem in $G$ is perturbed by imposing additional boundary conditions on the boundary $\Gamma_{\varepsilon}$ of $\Omega_{\varepsilon}$. A. M. Ilin constrúcted representations of such perturbed solutions $u_{\varepsilon}$ in $G_{\varepsilon}{ }^{\prime}=G \backslash \bar{\Omega}$, which are asymptotic with respect to ${ }^{\prime}$ tending to zero (e.g: [4-6]): A simplified method for simpler but most important cases in applications was proposed by the author in [2]. Also numerical solutions have been given for spe: cial cases (e.g. [8]).

Up to now, however, concerning the local asymptotic behaviour, apparently only fixed boundary conditions have been considered at the boundary of the hole, though transition (coupling) conditions could also be significant. We imagine, e.g., a temperature field in a domain occupied by a body with thermal conductivity $\lambda^{+}$ which contains but a little inclusion $\Omega_{\varepsilon}$ filled with material of another conductibility $\lambda-$ Therefore we shall describe, by means of the first stages of approximation, a method to construct an asymptotic expansion, above all, for a three-dimensional model problem - Poisson's equation; further it is shown that the method will also work in the two-dimensional case and, on principle; for more general equations. Finally, in , the special case of a spherical inclusion, a more direct procedure can be used, taking its pattern from $[2,3]$. - For proving the asymptotic character of the expansions obtained a variant of the maximum principle is applied which admits jumps of the first derivatives.

It should be remarked that there are investigations of related problems for, e. $g$., bodies including thin sheets with material constants growing to infinity while their 
thickness tends to zero (e.g.; $[1,8])$; the very interesting results consist in global; rather weak convergence statements, but without precise description of the local behaviour, and, of course, the methods used are quite different from ours.

\section{Formulation of the model problem}

Let $G \subseteq \mathbb{R}^{3}$ be a bounded domain containing the origin 0 , and $h$ a smooth function defined in $\bar{G}$. The boundary $S \doteq \partial G$ of $G$ should be smooth enough to permit classical solutions of the Dirichlet problem.

$$
\because G:-\Delta w=h(x), \quad S: w=g(x)
$$

for continuous boundary vialues $g$. Let, further, $\Omega$ be another bounded domain in $\mathbb{R}^{3}$ containing 0 , with smooth boundary $\Gamma=\partial \Omega$. Set $\Omega_{\varepsilon}=\varepsilon \Omega=\{x: x / \varepsilon \in \Omega\}, \Gamma_{e}=\varepsilon \Gamma$ $=\partial \Omega_{\varepsilon}$, and $G_{\varepsilon} \doteq G \backslash \bar{\Omega}_{\ell}$, which is a domain with a small hole (provided $\varepsilon$ is sufficiently i small). (1) can be regarded as a system governing a stationary temperature distribution $w$ in a body with constant thermal conductivity $\lambda^{+}=1$. Now, within the subset; $\Omega_{\varepsilon}$, the-material is displaced by another one with different conductibility $\lambda^{-}=\lambda>0$. In that way the temperature will vary, at least locally; the perturbed temperature $u=u_{\varepsilon}$ is subject to the conditions

$$
\begin{aligned}
& G_{\varepsilon}:-\Delta u=h(x), \quad S: u=g(x), \\
& \dot{\Omega}_{\varepsilon}:-\lambda \Delta^{\prime} u=h(x), \quad . \\
& \Gamma_{\varepsilon}: \bar{u}^{-}-u^{+}=0, \quad l(u) \doteq 0,
\end{aligned}
$$

$$
u=u_{\varepsilon}(x)= \begin{cases}u^{+}(x) & \text { for } \quad x \in G_{\varepsilon}, \\ u^{-}(x) & \text { for } \quad x \in \Omega_{\varepsilon}^{\prime}, \quad l(u)=\lambda \frac{\partial u^{-}}{\partial n}-\frac{\partial u^{+}}{\partial n}\end{cases}
$$

$n$ denotes the outer normal with respect to $\Omega_{\varepsilon}$. (For existence confer, e.g., [9].)

\section{Construction of an asymptotic expansion}

We set $u=w+v$ and ask for an asymptotic expansion, for $\varepsilon \rightarrow+0$, of the difference $v$ which is solution of the following problem:

$$
\begin{aligned}
& G_{\varepsilon}:-\Delta v=0, ' S: v=0, \\
& \Omega_{\varepsilon}:-\lambda \Delta v=(\lambda-1) \Delta w=(1-\lambda) h, \\
& \Gamma_{\varepsilon}: v^{-}-v^{+}=0, \cdot l(v)=(1-\lambda) \partial w / \partial n .
\end{aligned}
$$

Now the boundary condition on $S$ is omitted, we only demand the (extended) solution to be regular at infinity. So the exact difference $v$ is changed to an approximation $\bar{v}$. Next the two inhomógenities in (3) are removed for a jump of $\bar{v}$ along $\Gamma_{\varepsilon}$ by

$$
\bar{v}=v_{0}+\hat{w}, \quad \hat{w}(x)= \begin{cases}0 & \text { for } x \in G_{\varepsilon}, \\ (1-\lambda) \lambda^{-1} w(x) & \text { for } x \in \Omega_{\varepsilon},\end{cases}
$$

after which

$$
\begin{aligned}
& G_{\varepsilon}:-\Delta v_{0} \stackrel{+}{=} 0, \quad \Omega_{\varepsilon}:-\Delta v_{0}=0 \\
& \Gamma_{\varepsilon}: v_{0}^{-}-v_{0}^{+}=(\lambda-1) \lambda^{-1} w, \quad l\left(v_{0}\right)=0
\end{aligned}
$$


and $v_{0}(\infty)=0$. As third step we set

$$
v_{0}(x)=\left\{\begin{array}{lll}
W[\psi](x) & \text { for } & x \in G_{e} \\
\lambda^{-1} W[\psi](x) & \text { for } & x \in \Omega_{e}
\end{array}\right.
$$

where $W[\psi]$ denotes the double layer potential with density $\psi$ on $\Gamma_{e}$, so that all conditions of (5), except the third one, will be satisfied automatically. As it will be more favourable for the following, we introduce the stretched variables

$$
\xi=x / \varepsilon
$$

and, corresponding, $|\xi| \doteq \varrho=r / \varepsilon=|x| / \dot{\varepsilon}$, so that the potential $W$ is now given on the fixed boundary $\Gamma$ of the prototype domain $\Omega$ by

$$
W[\varphi](\xi)=\oiint_{\Gamma} \varphi(\tau) \partial \varrho^{-1} / \partial n_{\mathrm{r}} d \sigma_{\mathrm{r}},
$$

where $\varphi(\tau)=\psi(\varepsilon \tau)$. Adequately, we also rewrite the function $v_{0}$ as a function of $\xi$, too: $v_{0}(\xi)=v_{0}(x / \varepsilon)$. Using the jump property of $W$ at $\Gamma$

$$
W^{+}(\xi)=2 \pi \varphi(\xi)+W(\xi), W=(\xi)=-2 \pi \varphi(\xi)+W(\xi),
$$

where $W^{+}, W^{-}$are the limits from-the outside and from the inside, respectively, while $W(\xi)$ denotes the value on $\Gamma$ itself, we obtain the integral equation for the density $\varphi$,

$$
2 \pi \varphi(\xi)-\frac{1-\lambda}{1+\lambda} \dot{W}[\varphi](\xi)=\frac{\dot{1}-\lambda}{1+\lambda} w(\varepsilon \xi)
$$

on the surface $\Gamma$, with weakly singular kernel. Because the homogeneous adjoint integral equation turns out to provide, but as a single layer potential $V$, a solution of (5) .with $w$ replaced by 0 , regular at infinity, from the lemma below (maximum principle) it will follow that this solution is necessarily the trivial one. Therefore (8) will always be solvable; the solution will depend continuously on the right-hand side. Since a double layer potential will decrease for $\varrho=|\xi| \rightarrow \infty$, according to $\varrho^{-2}$, we obtain for

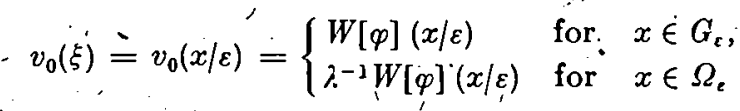

the estimate

$$
\left|\dot{v}_{0}(x / \varepsilon)\right| \leqq \dot{C} \varepsilon^{2} / r^{2}
$$

with a constant $C$ independent of $\varepsilon$, for $\varphi$ will be bounded from the uniform boundedness of the right-hand side of (8). With the function $v_{0}$ just constructed the approximate difference $\bar{v}$ in (4) satifies the conditions (3) exactly except that on $S$ : there will be $\bar{v}=v_{0}=O\left(\varepsilon^{2}\right)$. By the lemma already mentioned it' will turn out that

$$
u(x)=\bar{w}(x)+\hat{w}(x)+v_{0}(x / \varepsilon)+\varepsilon^{2} z_{1}(x)
$$

with $z_{1}$ bounded in $G$, independently of $\varepsilon$.

The further procedure in order to get higher approximations is apparent: The function $z_{1}$ will be expanded

$$
z_{1}=w_{1}+\hat{w}_{1}+v_{1}+\varepsilon^{2} z_{2}
$$

where $w_{1}$ is the solution of (1) with $h=0, g=-v_{0} / \varepsilon^{2}$ (in order to compensate the deviation of the boundary values on $S$ induced by $v_{0}$ ), $\hat{w}_{1}$ is defined analogously by 
(4) with $w_{1}$ instead of $w$, and $v_{1}$ is obtained by (5), (8), and (9) with $\dot{w}$.replaced by $w_{1}$ just as done for $v_{0}$, and so 'on. Sümming up we have

Theorem 1: The solution $u$ of the perturbed problem (2) admits an asymptotic expansion

$$
u(x)=\sum_{k=0}^{m} \varepsilon^{2 k}\left(w_{k}(x)+\hat{w}_{k}(x)+v_{k}(x / \varepsilon)\right)^{\prime}+\varepsilon^{2(m+1)} z_{m+1}(x)
$$

where $w_{0}=w$ is the solution of the unperturbed problem $(1), w_{k}(k \geqq 1)$ the solution of (1) with $h=0, g=-v_{k-1} / \varepsilon^{2}$, .

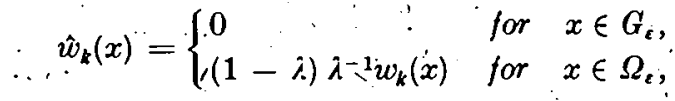

$$
\begin{aligned}
& v_{k}(\xi)= \begin{cases}W(\xi) & \text { for } \varepsilon \xi^{\prime} \in G_{e}, \\
\lambda^{-1} W(\xi) & \text { for } \varepsilon \xi \in \Omega_{\varepsilon},\end{cases}
\end{aligned}
$$

$W \stackrel{\prime}{=}\left[\varphi_{k}\right]$ the double layer potential on $\Gamma$ with density $\varphi_{k}$ which is a solution of the integral equation

$$
2 \pi \varphi_{k}(\xi)-\frac{1-\lambda}{1+\lambda} W\left[\varphi_{k}\right](\xi)=\frac{1-\lambda}{1+\lambda} w_{k}(\varepsilon \xi), \quad \xi \in \Gamma,
$$

and $z_{m+1}$ is bounded in all of $G$, independently of $\varepsilon$.

Re mark: The functions $v_{k}$ depend continuously on $\varepsilon$ because the corresponding solutions $\varphi_{k}$ of the integral equation (8) will do. Of course one could expand the right-hand side of (8) and obtain, for each $v_{k}^{\prime}$, an expansion with powers of $\varepsilon$, the coefficient functions depending on $\xi=x / \varepsilon$ only.

\section{The maximum principle}

In order to estimate the remainder $z_{m+1}$ of the expansion (13), and for the proof of solvability of the integral equation (8), we use the following variant of the maximum principle.

Lemma: Let $z, Z \in C(\bar{G}) \cap C^{2}\left(G_{\varepsilon}\right) \cap C^{1}\left(\bar{G}_{\varepsilon}\right) \cap C^{2}\left(\Omega_{\varepsilon}\right), \cap \dot{C^{\prime}}\left(\bar{\Omega}_{\varepsilon}\right)$,

$$
L^{ \pm} u=-\lambda^{ \pm} \cdot \Delta u+c(x) u, . l u=\lambda^{-} \frac{\partial u^{-}}{\partial n^{i}}-\lambda^{+} \frac{\partial u^{+}}{\partial n}
$$

where $c \in C(\bar{G})$ is nonnegative, $\lambda^{-}, \lambda^{+}$are positive constants, $n$ is the outside normal (with respect to $\Omega_{\varepsilon}$ ). If

$$
\begin{aligned}
& G_{\varepsilon}:\left|L^{+} z\right| \leqq L^{+} Z, \quad S:|z| \leqq Z, \\
& \Omega_{\varepsilon}:\left|L^{-} z\right| \leqq L^{-} Z, \quad \Gamma_{\varsigma}:|l z| \leqq l Z,
\end{aligned}
$$

then $|z| \leqq Z$ in all of $G$.

The proof consists in the usual application of the following proposition to the differences $v= \pm z-Z$, with apparently corresponding notations:'

Let $G_{1} \subseteq G_{2} \subseteq \cdots \subseteq G_{m}^{\prime}=G$ be bounded domains in $\mathbb{R}^{n}$ with smooth boundaries $S_{i}=\partial G_{i}$ which do not intersect each other. In the closure of each $D_{i}=G_{i} \backslash \bar{G}_{i-1}$ $\left(i=1 ; \ldots, m ; G_{0}\right.$ empty set) a linear uniformly elliptic positive operator $L_{i}$ of second order, with bounded coefficients, is defined for which the strong maximum principle 
is valid. Let $v$ be continuous in $\bar{G}$, with $v \leqq 0$ on $S=S_{m}=\partial G, v(x)=v_{i}(x)$ for $x \in \bar{D}_{i}$, and, in each $D_{i}$, be a classical solution of $L_{i} v_{i} \leqq 0$ with first derivatives continuous up to the boundaries. In any point of a boundary $S_{i}$ separating $D_{i}$ and $D_{i+1}$, for the outer normal derivatives the transition condition shall be fulfilled:

$$
\text { If } \partial v_{i+1} / \partial n<0 \text {, then } \partial v_{i} / \partial n<0 \text {. }
$$

Then $\tilde{v}$ cannot assume a positive maximum in the interior of $G$. Apparently it is only , to be shown that $v$ cannbt attain a positive maximum at a point $x_{i}$ of a boundary $S_{i}(i \leqq m-1)$. But if this should be the case, and $v_{i+1}$ not a constant, then $\partial v_{i+1} / \partial n$ $<0$ in $x_{i}$, and $\left(^{*}\right)$ would entail still greater values of $v=v_{i}$ in $D_{i}$. If $v_{i+1}=$ const, the árgument may be repeated with respect to the boundary $S_{i+1}$, and so on

Supplement: The assertion of the lemma will be maintained if there is a surface (e.g., a sphère) in $G$, where $Z$ has only continuous derivatives of first order.

In order to apply the lemma to prove that $z_{1}$ in (11) is bounded we state the continuity of $z_{1}$ at $\Gamma_{\varepsilon}$ and

$$
\begin{aligned}
& G_{\varepsilon}:-\Delta z_{1}=0, \quad S:\left|z_{1}\right|=\left|-v_{0}\right| \varepsilon^{2} \mid \leqq C, \\
& \Omega_{\varepsilon}:-\lambda \Delta z_{1}=0, \quad \Gamma_{\varepsilon}: l z_{1}=0 .
\end{aligned}
$$

Apparently $\bar{Z}=C=$ const will be an upper bound for $\left|z_{1}\right|$. Preceding a step $(m=1)$ we have to estimate $z_{2}$ from (12). Inserting the definitions or properties of $w_{1}, \hat{w}_{1}, v_{1}$, and $z_{1}$ we see that $z_{2}$ is continuous in $G$ and satisfies a system like (14), with $v_{0}$ replaced by $v_{1}$. Thus, 'by induction, the general assertion can be proved.

As to the solvability of the integral equation (8); it is to be shown that a solution of (5), with $w=0$, represented by a single layer potential, must vánish identically. For this sake, the domain is extended to a ball of arbitrary radius; on its surface the potential, will be as small as one likes and, according to the lemma, also in the ball and in the included domain $G$.

\section{The two-dimensional case}

The assertions of Theorem 1 will apparently be maintained, with obvious modifications, for space dimensions greater than three. Also fọ̀ dimension two one expects, for the present, the formal expansion procedure to run quite similar: In the expansion (13) the powers $\varepsilon^{2 k}$ are to be replaced by $\varepsilon^{k}-$ corresponding to the fact that the $v_{k}(\xi)$, given by double layer potentials, will now decrease with order $O\left(|\xi|^{-1}\right)$ only - and in the integral equation (8) $2 \pi$ is substituted by $\pi$. Further on, the existence of a uniform bound for $z_{m+1}$ in the remainder $\varepsilon^{m+1} z_{m+1}$-can be proved as just done by the lemma. But it cannot be applied immediately, as at the end of Section 3, to the proof - of the solvability of (8): The function $v$ satisfying (5) (with $w=0$ ), since generated by. a single layer, cannot be supposed here, a ’priori, to tend to zero at infinity, which . had been essential above.

But we cañ conclude as follows: If $v(\xi)=V[\bar{\chi}](\xi)$ is a single layer potential which will.fulfil $l v=0$ at $\Gamma$ for a solution $\chi$ of the adjoint equation $x \chi-(1-\lambda)(1+\lambda)^{-1}$ $\times \partial V[\chi] / \partial n=0$, then

$$
\vartheta(\xi)=\left\{\begin{array}{lll}
v(\xi)+W[\varphi](\xi) & \text { for } & \bar{\xi} \notin \Omega, \\
\lambda v(\xi)+W[\varphi](\xi) . & \text { for } & \xi \in \Omega
\end{array}\right.
$$

with the double layer potential $W[\varphi], \varphi \varphi-(1 / 2 \dot{\pi})(1-\lambda) v$, passes $\Gamma$ cońtinuously including its first derivatives. Therefore $v$ is a potential function in all the plane $\mathbb{R}^{2}$, 
with logarithmic growth at infinity, at most, but.bounded from above or below - and such a singularity must be, a removable one: $v=c=$ const: Now (15) shows that $v(\xi)=c+O\left(|\xi|^{-1}\right)$ as $|\xi| \rightarrow \infty$, and by the lemma $v(\xi)-c \equiv 0$ will follow. Especially, $\partial v / \partial n=0$ on $\Gamma_{\varepsilon}$ and, therefore, $\chi=0$ guarantees solvability of the integral equation (8) also in this case.

Theorem 2: In a plane domain $G$ with inclusion $\Omega_{\varepsilon}$ the solution $u$ of the perturbed problem (2) admits an asymptotic expansion

$$
u=\sum_{k=0}^{m}\left(w_{k}+\dot{w}_{k}+v_{k}\right) \bar{\varepsilon}^{k}+\dot{\varepsilon}^{m+1} z_{m+1}
$$

with functions $w_{k}, \hat{w}_{k}, v_{k}$ defined like in Theorem 1 , but $w_{k}(k \geqq 1)$ solution of (1) with $g=-v_{k-1} / \varepsilon$ and $v_{k}(\xi)=v_{k}(x / \varepsilon)=W\left[\varphi_{k}\right](\xi)$ given by the integral equation on $\Gamma$

$$
\pi \varphi_{k}(\xi)-\frac{1-\lambda}{1+\lambda} W\left[\varphi_{k}\right](\xi)=\frac{1-\lambda}{1+\lambda} w_{k}(\varepsilon \xi)
$$

\section{Remarks on the case $-\Delta+c$}

The problem will now read "

$$
G:-\Delta w+c(x) w=h(x), \quad S: w_{1} \doteq g(x) .
$$

and the perturbed problem is

$$
\begin{aligned}
& G_{\varepsilon}:-\Delta \dot{u}+c(x) u=h(x), \quad S: u=g(x), \\
& \Omega_{\varepsilon}:-\lambda \Delta u+c(x) u \doteq h(x), \quad \Gamma_{\varepsilon}: u^{-}-u^{+}=0, \quad l u=0,
\end{aligned}
$$

with a nonnegative continuous function $c$ in $\bar{G}, G, \Omega$ are bounded domains in $\mathbb{R}^{3}$ as in Section $1, \Omega_{\varepsilon}=\varepsilon \dot{\Omega}, \Gamma_{\varepsilon}=\varepsilon \Gamma=\partial \Omega_{\varepsilon}$. In addition, for technical reasons, $\Omega$ is to be supposed star-shaped with respect to a ball centered at the origin. As leading terms of an asymptotic approximation, the same functions as defined in (4), (9) for the simpler model above turn out to be also suited in this case. The difference $v=u-w$. will here satisfy the conditions corresponding to $(3)$ - the differential equations in. $G_{\xi}$ and $\Omega_{\varepsilon}$ augmented by $c(x) v$ on the left-hand side. But as approximating difference $\bar{v}$ we will again choose the solution, regular at infinity, of .

$$
\begin{aligned}
& \mathbb{R}^{3} \backslash \bar{\Omega}_{\varepsilon}:-\Delta \bar{v}=0, \quad \Omega_{\varepsilon}:-\lambda \Delta \bar{v}=(\lambda-1) \Delta w \\
& \Gamma_{\varepsilon}: \bar{v}^{-}-\bar{v}^{+}=0, \quad i \bar{v}=(1-\lambda) \partial w / \partial n . .
\end{aligned}
$$

Therefore we set, combining (4) and (9), ।

$$
\dot{v}(\dot{x})=\hat{w}(x)+v_{0}(x / \varepsilon)=\left\{\begin{array}{l}
W[\varphi](x / \varepsilon) \\
(1-\lambda) \lambda^{-1} w(x)+\lambda^{-1} W[\varphi](x / \varepsilon)
\end{array}\right.
$$

for $x \in G_{\varepsilon}$ or $x \in \Omega_{\varepsilon}$, respectively, where $\varphi$ is the solution of the integral equation (8). The remainder $z_{0}$ in $u=w+\hat{w}+v_{0}+z_{0}$ will now be submitted to

$$
\begin{aligned}
& G_{\varepsilon}:-\Delta z_{0}+c z_{0}=-c v_{0}, \quad S: z=-v_{0}, \\
& \Omega_{\varepsilon}:-\lambda \Delta z_{0}+c z_{0}=-c\left[(1-\lambda), \lambda^{-1} w+v_{0}\right], \\
& \Gamma_{\varepsilon}: z_{0}^{-}-z_{0}^{+}=0, \quad l z_{0}=0 .
\end{aligned}
$$


As $v_{0}$ is given by a double layer potential $W$ with bounded density, we will have '

$$
\begin{array}{ll}
\left|-c v_{0}\right| \leqq C(\varepsilon / r)^{2} & \text { in } G_{e}, \\
\mid-c\left[(1-\lambda)^{1} \lambda^{-1} w+\dot{v}_{0}\right] \mathrm{S} \leqq C \text { in } \Omega_{\varepsilon}, \\
\left|-v_{0}\right| \leqq C \varepsilon^{2}
\end{array}
$$

If $\dot{G}, \Omega_{\varepsilon}$ are contained in balls of radii $R$ and - e.g. $-\dot{2} \varepsilon$, then

$$
Z_{v}= \begin{cases}C \varepsilon^{2} \ln (R / r) & \text { for } r>2 \varepsilon \\ C\left[\varepsilon^{2} \ln (R / 2 \varepsilon)+1 / 2\left(4 \varepsilon^{2}-r^{2}\right)\right] . & \text { for } r<2 \varepsilon\end{cases}
$$

is continuously differentiable in $\mathbb{R}^{3}$ and, for suitable constant $C$, will majorize $z_{0}$ in the sense of the lemma, possibly with exception of the last condition on $\Gamma_{\ell}$ :

$$
\left|l z_{0}\right|=0 \leqq l z=C(1-\lambda) \varepsilon^{2} r^{-1} s(x)
$$

where $s(x)$, denotes the scalar product between the outer unit normal $n$ at $x \in \Gamma_{\varepsilon}$ and the normed radius vector $\overrightarrow{0} \vec{x} / r$. As $\Omega$ is assumed star-shaped, $s(\dot{x})$ will be strictly positive on $\Gamma_{c} ;$ and therefore the lemma can be applied if $\lambda \leqq 1$.

The case $\lambda>1$ requires a modification. Let $q$ be the harmonic function defined outside $\Omega$, regúlar at infinity, and equal to 1 on $\Gamma$. There exists a positive constant $c_{0}$ so that, on $\Gamma, \partial \dot{q} / \partial n \leqq-c_{0}$. Then

$$
Q(x)=\left\{\begin{array}{lll}
g(x / \varepsilon) & \text { for } & x \in \mathbb{R}^{3} \backslash \Omega_{e} \\
1 & \text { for } & x \in \Omega_{e}
\end{array}\right.
$$

has the properties $\Delta Q=0$ in $\Omega_{\varepsilon}$ and outside $\Omega_{e}, Q>\dot{0}$, bounded, and $l Q \geqq c_{0} / \varepsilon$. Therefore $Z^{\prime}=Z+c_{1} \varepsilon^{2} Q$ with $Z$ from (22) has all majorizing properties claimed by the lemma, especially.

$$
l Z^{\prime} \geqq\left(4 C(1-\lambda) \varepsilon^{2} / r+c_{0} c_{1} \varepsilon\right) s(x) \geqq 0
$$

if $c_{i} \geqq 4(\lambda-1) C / r_{0}$, where $r_{0}$ is the radius of a ball, centered at 0 , which is contained in $\Omega$. Thus we obtain, in either case,

$$
\left|z_{0}\right| \leqq C \varepsilon^{2}|\ln \varepsilon|
$$

In order to raise the degree of approximation, next we have to expand $z_{0}=w_{1}+\bar{v}_{1}$ $+z_{1}$, where $w_{1}$ is the solution of an unperturbed problem which compensates the values of $v_{0}$ on the outer boundary $S$. Looking at the problem (20) for $z_{0}$ we see that it can be interpreted as perturbation (like (2) in proportion to (1)) of the problem

$$
G:-\Delta \dot{w}_{1}+c w_{1}=-c\left(v_{0}+\hat{w}\right), \quad S: w_{1}=-v_{0}
$$

Therefore the cónstruction just carried out can be repeated, with $-c\left(v_{0}+\hat{w}\right)$ instead of $h,-v_{0}$ instead of $g$, and in (18), (19) $\bar{v}, w, \hat{w} ; v_{0}$ replaced by $\bar{v}_{1}, w_{1}, \hat{w}_{1}$ (cf. (4)), $v_{1}$, respectively. For the remainder $z_{1}$ in $z_{0}=w_{1}+w_{1}+v_{1}+z_{1}$ we now obtain, analogously, again (20), with $z_{1}, v_{1}, w_{1}$ instead of $z_{0}, v_{0}, w_{0}=w$. As the estimate (23) for $z_{0}$ will also hold, the more, for $w_{1}$ (using the lemma with $\lambda^{-}=\lambda^{+}=1$ ), the right-hand sides of (18), (19), and (8), rewritten with the new quantities, will be of order $O\left(\dot{\varepsilon}^{2}|\ln \varepsilon|\right)$, the estimation above, applied to $z_{1}$, yields

$$
\left|z_{1}\right| \leqq C \dot{\varepsilon}^{4}(\ln \varepsilon)^{2}
$$


Proposition 1: The solution $u$ of the perturbed problem (17) admits expansions

$$
\begin{aligned}
u & =w_{0}+\hat{w}_{0}+v_{0}+z_{0} \\
& =w_{0}+\hat{w}_{0}+v_{0}+w_{1}+\hat{w}_{1}+v_{1}+z_{1} .
\end{aligned}
$$

with $w_{0}=w$ solution of the unperturbed problem $(16), \hat{w}_{0}+v_{0}$ and $\hat{w}_{1}+v_{1}(c f .(19))$ corrections; previously in the vicinity of $\Omega_{t}$, while $w_{1}$ (cf. (24)) corrects on $S$; the remainders $z_{0}, z_{1}$ are uniformly bounded according to (23), (25).

\section{Spherical inclusions ${ }^{-}$}

Finally, it should be remarked that in the special case where $\Omega_{\varepsilon}$ is a ball $K_{\varepsilon}$ of radius $\varepsilon$, centered at 0 , an asymptotic expansion of the solution of the model problem (2) would better be established more directly; based on the Taylor expansion of the unperturbed solution $w$, at $x=.0$,

$$
w=w^{0}+w_{i}^{0} x_{i}+\frac{1}{2 !} w_{i j}^{0} x_{i} x_{j}+\cdots,
$$

where $w_{i}^{0}=\partial w / \partial x_{i}$, taken at $x=0$, and analogously. As a first approximation to the equations (3) for the difference $v=u-w$ turns out to be reasonable, with regard to the estimation of the remainder,

$$
\begin{aligned}
& G_{e}: \quad-\Delta \tilde{v}=0 \\
& K_{\epsilon}:-\lambda \Delta \tilde{v}=(1-\lambda) h(0)=(\lambda-1) \Delta u(0) \\
& \partial K_{\varepsilon}: \tilde{v}^{-}-\bar{v}^{+}=0, \quad l \tilde{v}=(\lambda-1)\left[w_{i}^{0} x_{i} / \ddot{r}+w_{i j}^{0} x_{i} x_{j} / r\right]
\end{aligned}
$$

with $\tilde{v}(\infty)=0$. Suitable expansion functions will be, for $\bar{v}=\bar{v}^{+}$in $G_{\varepsilon}$, linear combinations of derivatives of the principal solution, and for $\tilde{v}=\bar{v}^{-}$in the ball $\tilde{K}_{\varepsilon}$, homogeneous polynomials, some of which solve the approximating equation on $K_{\varepsilon}$, and the other ones are harmonic. For the latter it is best to take those which arise in the nominators $H(x)$ of the derivatives just mentioned if they are represented by $H(x) / r^{m}$. After inserting these ansat $\%$ es into (26) and equating at $r=\varepsilon$ the polynomials, or nominator polynomials, respectively, of the same order, some calculations will provide, the exact solution of (26), in the three-dimensional case:

$$
\therefore \quad\left\{\begin{array}{l}
\bar{v}^{+}=\frac{1-\lambda}{3+2 \lambda}\left[\frac{h(0)}{3} \varepsilon^{2} \bar{\varrho}^{3}+\frac{3+2 \lambda}{2+\lambda} \bar{\varrho}^{3} w_{i}^{0} x_{i}+\bar{\varrho}^{5} w_{i j}^{0} x_{i} x_{j}\right], \\
\tilde{v}^{-}=\frac{1-\lambda}{3+2 \lambda}\left[\frac{h(0)}{3} \varepsilon^{2}+\frac{3+2 \lambda}{2+\lambda} w_{i}^{0} x_{i}+u_{i j}^{0} x_{i} x_{i}+\frac{h(0)}{2 \lambda} \varepsilon^{2}\left(1-\bar{\varrho}^{2}\right)\right]
\end{array}\right.
$$

where $\bar{\varrho}=\varepsilon / r$. It should be pointed out that in $\bar{v}^{+}$the principal solution $1 / r$ does not appear itself. This will hold also for other dimensions; especially for $n=2$ no logarithmic term will trouble - and this will confirm the fact stated above, in Section 4 , that the expansion procedure will also run in the two-dimensional case.

In order to estimate the degree of approximation to $u$ given by $w+\bar{v}$ it is established, for $z=u-(w+\tilde{v})$,

$$
\left\{\begin{array}{l}
G_{\varepsilon} \vdots-\Delta z \doteq 0, \quad S: z=O\left(\varepsilon^{3}\right) \\
K_{\varepsilon}:-\lambda \Delta z=O(\varepsilon), \quad \partial K_{\varepsilon}: z^{-}-z^{+}=0, \quad l z=O\left(\varepsilon^{2}\right):
\end{array}\right.
$$


A majorant function $Z$ which satisfies the conditions of the lemma, except that on $S$, will be given by

$$
Z=\left\{\begin{array}{l}
Z^{+}=C_{0} \varepsilon^{4} / r \\
Z^{-}=1 / 6 C_{1} \varepsilon\left(\varepsilon^{2}-r^{2}\right)+C_{0} \varepsilon^{3}
\end{array}\right.
$$

with proper constants $C_{0}, C_{1}$. Merely to be able to apply the lemma; formally a function $\varepsilon^{3} w_{1}$ must be added to $\tilde{v}$, where

$$
G:-\Delta \dot{w}_{1}=0, \quad \cdot S: w_{1}=-\frac{1-\lambda}{2+\lambda} \frac{w_{i}^{0} x_{i}}{r^{3}}
$$

Then in (28) we will have $z=O\left(\varepsilon^{5}\right)^{\text {on }} S-$ the other.conditions are not concerned and $Z$ is now an admissible majorant function.

Proposition 2: In the case $n=3$ and if the incluision occupies the ball $K_{\varepsilon}$, an asymptotic expansion of the solution of (2) is

$$
u(x)=u(x)+\tilde{i}(x)+\varepsilon^{3} z_{1}(x, \varepsilon)
$$

with $\tilde{v}$ given by $(\dot{2} 7)$ and $\left|z_{1}(x, \varepsilon)\right| \leqq C \min \{\varepsilon / r, 1\}$

If higher approximations are, desired, then, of course, the function $w_{1}$ compensating on the outer boundary $S$, will be essential and must, further, be expanded itself 'similarly as $w$.

Remark: The limit case $\lambda=\infty$ where the-temperature $u$ must be constant + in $K_{e}$ has been treated in, $[2]$; the result there proves to be the limit, for $\lambda \rightarrow \infty$, of $(29)$, (27).

\section{REFERENCES}

[1] Acerbi, E., Buttazzo, G., and D. Percivale: Thin inclusions in linear elasticity: a variational approach. J. rëine angew. Math. 386i (1988), 99-115.

[2] GöhdE, D.: Singuliire Störung von Randwertproblemen' durch ein kleines Loch im Gebiet. Z. Anal. Anw. 4 (1985), 467-477.

[3] Görts, D.: Störung stationärer Temperaturfelder durch ḱleine Einschlüsse mit großer Wärmeleitfähigkeit. Wiss. Beiträge Ingenieurhochschule Zwickau 12 (1986) 5, $91-96$.

[4] Ильин, А. М.: Краевая задача для әллиптического уравненил второго порядка в области с узкой щелью. 1. Двумерный случал. Мат. Сӧорник.99 (1976), 514-537.

[5] Нльин, А. М.: Краевая задача для әллиптического уравнения второго порядка в области с узкоп щелью. 2. Область с малым отверстием. Мат. Сборник 103 (1977), $265-284$.

[6] Ильи, А. М.: Иссленование асимптотики решения јллиптической краевой задачи в области с.малым отверстием. Труды Сем. им. Гетровского (6 (1981), 57-82.

[7] Симоненко, И. Б.: Задачи әлектростатини в неонородной среде. Случай тонкого диэлектрика с больщой диолектрической постопнио̆ ї. Дифф. ур-ия 11 (i975), $1870-1878$. 
[8] Шишкин, Г.'И.: Решение краевой задачи для уравнения второго піорядка с малым отверстием в случае кругово с симметрии. В сб'е: Дифференцияльные уравнения с малым параметром. Свертловск: Уральский Научный Центр Акад. Наук СССР 1984 , стр. $104-118$.

[9] Wanka, G., und J. Wanka: Ein Existenztheorem für eine Klasse von allgemeinen Dirich. letschen Randkontaktproblemen bei linearen Differentialgleichungen zweiter Ordnung. Math. Na'chr. 110 (1983), 215-229.

Manuskripteingang: 18. 05. 1988; in revidierter Fassung 26. 08. 1988

\section{VERFASSER:}

Prof. Dr. Dietrich GöndF

Sektion Math./Naturwiss./Inf. der Technischen Hั̣ochschule

Dr.-Friedrichs-Ring $2 \mathrm{a}$

DDR.9541 Zwickau

Added in proof: Solving (8), at first, only for the constant term of Taylor's expansion of the right-hand side, the properties of double layer potential $W$, with constant density (Gauss' integral - e.g., vanishing outside $\Omega_{\varepsilon}$ !), will allow the constant $C$ in the basic estimate (10) to be replaced by $C \cdot \varepsilon$, so that all powers of $\varepsilon$, in the sequel; can be lifted respectively; especially, (13) is true even with $\varepsilon^{3 k}$ instead of $\varepsilon^{2 k}$, and in $\left(13^{\prime}\right) \varepsilon^{k}$ may be replaced by $\varepsilon^{2 k}$. 